die ätherlösliche Lezithinglukose ( $\mathrm{B}$ ing ${ }^{8}$ ). Eine Erklärung für diese Erscheinung bot die Beobachtung, daß Aether bei Gegenwart von Lezithin imstande ist, erhebliche Mengen von Wasser aufzunehmen. Dies kann durch nachstehenden einfachen Versuch leicht demonstriert werden.

In zwei graduierte Meßzylinder mit eingeschliffenem Glasstopfen kommt je $15 \mathrm{ccm}$ Wasser und $15 \mathrm{ccm}$ trockener Aether. $\mathrm{Zu}$ der einen Probe kommt noch $0,3 \mathrm{~g}$ getrocknetes Lezithin, Hierauf wird wiederholt durchgeschüttelt, dann bis zur scharfen Scheidung stehen gelassen und das Volumen der Schichten abgelesen. Die Aetherschicht der mit Lezithin versetzten Probe zeigt $14,2 \mathrm{ccm}$, die Wasserschicht $15 \mathrm{ccm}$. Bei Kontrollprobe ohne Lezithin zeigt die Aetherschicht $12,5 \mathrm{ccm}$, die Wasserschicht $16 \mathrm{ccm}$.

$\mathrm{DaB}$ das vom Aether aufgenommene Wasser an der Lossung von Salzen wesentlich beteiligt ist, zeigt die Beobachtung, daB das mit Salzen bzw. mit Zucker verriebene Lezithin sich in

8) Bing, Skandin. Arch. f. Physiol. 9, 336 (1899). Dieser Autor hat noch zahlreiche andere atherlosliche Lezithinverbindungen" dargestellt.
Aether erst lost, bis Wasser hinzugefügt ist, wie ja auch das Jekorin nur in wasserhaltigem Aether löslich ist. Daneben mag auch die Lipoidlöslichkeit von Salzen eine gewisse Rolle spielen, was daraus hervorgeht, dab alkohollösliche Salze $\left(\mathrm{CuCl}_{2}\right)$ in viel größerer Quantität von der ätherischen Lezithinlösung aufgenommen werden als alkoholunlösliche. Instruktiv ist z. B. ein Versuch mit $\mathrm{CuCl}_{2}$, dessen Aufnahme durch ätherische Lezithinlösung sich durch Grünfärbung der Lösung verrät.

Die hier beschriebene Eigenschaft ätherischer Lezithinlösung wird sich vielleicht zur Klärung der Jekorinfrage heranziehen lassen und möglicherweise noch andere Beobachtungen, die gelegentlich der Analyse von Organen und Organflüssigkeiten gemacht worden sind, dem Verständnis näher bringen.

Anhangsweise sei erwähnt, daß wir analoge Versuche wie mit Lezithinlösungen auch mit alkoholischen und atherischen Cholesterinlösungen ausgeführt haben. Diese Lösungen zeigten jedoch mit keinem der angewandten Reagenzien eine Zustandsänderung, was auch zu erwarten war, da das Cholesterin sich hier in echter Lösung befindet.

\title{
Ueber die antagonistische Wirkung der Neutralsalze auf die Quellung des Fibrins in Säuren und Alkalien.
}

\author{
Von Martin H. Fischer und Gertrude Moore.
}

(Aus dem Frank B. Yoakum-Laboratorium der Oakland School of medicine, California, U. S. A.)

Die im folgenden beschriebenen Versuche bilden eine Fortsetzung derjenigen, die bereits in zwei früheren Mitteilungen über die Quellung des Fibrins veröffentlicht wurden ${ }^{1}$ ).

Wir zeigten in diesen Abhandlungen, daB der Betrag der Quellung des Fibrins in Lösungen irgendwelcher Säuren und Alkalien beträchtlich herabgesetzt wird durch Zusatz irgendwelcher Neutralsalze, z. B. zu einer sauren Lösung. Je mehr von einem solchen Salz zu der sauren Lösung hinzugegeben wird, um so weniger wird das Fibrin in dieser Lösung quellen. Wir fanden indessen beim Vergleich der Wirkung verschiedener äquimolekularer Salzlösungen, daß die einzelnen Salze sich keineswegs gleichmäßig

1) Martin H. Fischer und Gertrude Moore, Ueber die Quellung des Fibrins, Amer. Joum. Physiol.20, 330 (1907). Martin H. F is cher, Weitere Versuche uber die Quellung des Fibrins, Pflï g er's Arch.f. ges. Physiol. 125, 99 (1908): siehe auch die Referate in Koll-Zeitschr. 5, 121 u.f. (1909). in bezug auf ihren quellungshemmenden Effekt verhielten. Bei vergleichenden Versuchsserien mit Reihen von Salzen, die ein gemeinsames Kation haben, zeigte sich, daß die Reihenfolge der Anionen immer die gleiche war. Umgekehrt fanden wir auch beim Vergleich von Salzen mit gemeinsamem Anion, daß die Reihenfolge der Kationen stets dieselbe war. Diese Resultate führten uns zu dem SchluB, daB di e quellungshindernde Wirkung irgend eines Salzes auf die $Q$ uellung des Fibrins in irgend einer Säre oder $B$ ase, wenigstens in der Hauptsache, der Ausdruck ist für die Summe der Einzelwirkungen der sie konstituierenden lonen.

Unsere früher publizierten Versuche gestatteten uns nur eine vorlăufige Liste der Anionen und Kationen entsprechend ihrer quellungshemmenden Wirkung auf Fibrin in saurer Lösung aufzustellen. Wir waren seitdem 
imstande, viele weitere Versuchsserien auszuführen. Auf Grund dieser können wir die folgenden Ionentabellen aufstellen, die, obschon sie nur unbedeutend von den früher veröffentlichten abweichen, insofern besser sind, als sie sich auf längere und öfter wiederholte Versuchsreihen gründen.

Die Anionen ordnen sich in folgender Reihe. Die Quellung des Fibrins in irgend einer Säure oder Base wird am wenigsten gehindert durch Zusatz des erstgenannten Anions; die quellungshemmende Wirkung der zugesetzten Anionen nimmt folgendermaßien zu:

Chlorid, Bromid, Nitrat, Sulfozyanat, Jodid, Azetat, Sulfat, Phosphat, Tartrat, Zitrat.

Die Kationenreihe ist folgende (das Kation mit dem geringsten quellungshemmenden Einflub ist zuerst genannt):

Kalium, Natrium, Ammonium, Magnesium, Barium, Kalzium, Kupfer, Eisen.

Unsere Versuchsmethode war ähnlich der in den früheren Abhandlungen beschriebenen. Gewogene Mengen $(0,25 \mathrm{~g})$ von sorgfältig gewaschenem, pulverisiertem Fibrin wurden in Reagenzgläser von gleichem Durchmesser $(2 \mathrm{~cm})$, die gemessene Mengen $(20 \mathrm{ccm})$ der entsprechenden Lösungen enthielten, hineingebracht. Nachdem das Fibrin sein Quellungsmaximum erreicht hatte, wurde die Höhe der Fibrinsäule in Millimetern gemessen. Um die Qellung zu erleichtern, wurden die Reagenzröhren gelegentlich geschüttelt. Um vergleichbare Resultate zu erhalten, wurden natürlich alle Röhren einer Versuchsreihe in genau gleicher Weise behandelt.

Die folgenden Tabellen mögen als Beispiele dafür dienen, wie die Daten, auf Grund welcher die obigen Schluffolgerungen gezogen wurden, erlangt wurden.

In Tabelle I kommen die Wirkungen verschiedener A $\mathrm{n}$ i o n en mit einem gemeinsamen Kation zum Vergleich. Bei Durchsicht müssen die Zahlenreihen abwärts gelesen werden, da jede Vertikalreihe eine Versuchsreihe darstellt, die unter genau gleichen äuBeren Bedingungen (bzw. Schütteln, Temperatur usw.) vorgenommen wurde usw. Die unteren Horizontalreihen zeigen die Größe der Versuchsfehler. Die Ablesungen in allen Tabellen wurden nach 24 Stunden gemacht.

Tabelle I

Durchmesser der Reagenzröhren: $2 \mathrm{~cm}$. Fibrinmenge: $0,25 \mathrm{~g}$.

Konzentration der Lösung: 1/40 mol. Salz in $1 / 40$ norm. Salzsäure.

\begin{tabular}{|c|c|c|c|c|c|c|c|c|c|}
\hline & Kalium & Natrium & $\begin{array}{c}\text { Ammo- } \\
\text { nium }\end{array}$ & $\begin{array}{c}\text { Magne- } \\
\text { sium }\end{array}$ & Barium & Kalzium & Strontium & Kupfer & Eisen \\
\hline $\begin{array}{l}\text { Chlorid } \\
\text { Bromid } \\
\text { Nitrat }\end{array}$ & $\begin{array}{l}23 \\
21 \\
18\end{array}$ & $\begin{array}{l}24 \\
22 \\
18\end{array}$ & $\begin{array}{l}22 \\
19 \\
18\end{array}$ & $\begin{array}{l}18 \\
16 \\
12\end{array}$ & $\begin{array}{l}17 \\
14 \\
13\end{array}$ & $\begin{array}{l}18 \\
16 \\
13\end{array}$ & $\begin{array}{l}17,5 \\
15 \\
12\end{array}$ & $\begin{array}{l}19 \\
-\end{array}$ & $\frac{14}{9}$ \\
\hline $\begin{array}{l}\text { Sulfozyanat } \\
\text { Jodid } \\
\text { Azetat }\end{array}$ & $\frac{13}{12}$ & $\overline{11,5}$ & $\overline{-}$ & $\frac{-}{6(?)}$ & $\frac{11}{7}$ & - & - & - & - \\
\hline $\begin{array}{l}\text { Sulfat } \\
\text { Phosphat } \\
\text { Zitrat }\end{array}$ & $\frac{8}{7}$ & $\begin{array}{l}8,5 \\
8 \\
8\end{array}$ & $\begin{array}{r}7 \\
- \\
-\end{array}$ & $\begin{array}{r}8 \\
- \\
-\end{array}$ & - & - & - & $\begin{array}{l}8 \\
- \\
\end{array}$ & - \\
\hline $\begin{array}{c}1 / 40 \text { norm. } \\
\mathrm{HCl} \\
\text { Destilliertes } \\
\text { Wasser }\end{array}$ & 44 & 44 & 40 & $40^{\circ}$ & 45 & 40 & 44 & $\begin{array}{r}45 \\
7\end{array}$ & $\begin{array}{l}400 \\
7\end{array}$ \\
\hline
\end{tabular}

In Tabelle II kommen die verschiedenen Kation en mit gleichem Anion bezüglich ihrer quellungshemmenden Wirkung auf Fibrin in
Salzsäure zum Vergleich. Die Zahlenreihen müssen wieder $a b$ ärts betrachtet werden. - Tabelle III zeigt die quellungshemmenden 
Tabelle II

Durchmesser der Reagenzröhren: $2 \mathrm{~cm}$. Fibrinmenge: $0,25 \mathrm{~g}$

Konzentration der Lösung: 1/40 mol. Salz in 1/40 norm. Salzsäure.

\begin{tabular}{l||c|c|c||c|c||c|c}
\hline & Chlorid & Bromid & Nitrat & Jodid & Azetat & Sulfat & Zitrat \\
\hline \hline Kalium & 23 & 21 & 18 & 16 & 11 & 6 & 6 \\
Natrium & 21 & 20 & $16(?)$ & 15 & 10 & 7 & 6 \\
Ammonium & 20 & 18 & 18 & 15 & 10 & 5 & - \\
\hline Magnesium & 18 & 14 & 15 & 13 & 7 & 5 & - \\
Barium & 18 & 14 & 14 & 14 & $11(?)$ & 8 & - \\
Kalzium & 17 & 14 & 11 & 9 & 7 & - & - \\
Strontium & 18 & 13 & 12 & 10 & - & - & - \\
\hline Kupfer & 18 & - & - & - & - & 6 & - \\
Eisen & 15 & - & 10 & - & - & - & - \\
\hline 1/40 norm. & & & & & & & \\
$\quad$ HCl & 40 & 40 & 40 & 40 & 40 & 40 & 42 \\
Destilliertes & 7 & 7 & 7 & 7 & 7 & 7 & 7 \\
$\quad$ Wasser & 7 & 7 & & 7 & \\
\hline
\end{tabular}

Tabelle III

Durchmesser der Reagenzröhren: $2 \mathrm{~cm}$. Fibrinmenge: $0,25 \mathrm{~g}$.

Konzentration der Lösung: $1 / 40 \mathrm{~mol}$. Salz in $1 / 40$ norm. Natronlauge.

\begin{tabular}{l||c|c|c|}
\hline & Kalium & Natrium & Ammonium \\
\hline \hline Chlorid & 33 & 31 & 8 \\
Bromid & 32 & 29 & 8 \\
Nitrat & 31 & 28 & 8 \\
\hline Sulfozyanat & 28 & - & - \\
Azetat & 29 & 23 & 8 \\
\hline \begin{tabular}{l||c|c|} 
Sulfat \\
Phosphat
\end{tabular} & 24 & 23 & 7 \\
Zitrat & 25 & 22 & - \\
\hline $\begin{array}{l}\text { 1/40 norm. } \\
\text { Na OH }\end{array}$ & 55 & 56 & 55 \\
$\begin{array}{l}\text { Destilliertes } \\
\text { Wasser }\end{array}$ & 7 & 7 & 7
\end{tabular}

Wirkungen verschiedener Salze auf Fibrin in der Lösung einer Base. Da eine große Zahl der Hydroxyde sehr unlöslich ist, konnte nur eine begrenzte Gruppe von Salzen verglichen werden. Durch A bwärtslesen der Zahlen können die Anionen, durch Seitwärtslesen die Kationen miteinander verglichen werden.

Die Ammoniumsalzreihe ist nicht ohne Interesse darum, weil die Zahlen alle niedrig sind. Um dies $z u$ erklären, brauchen wir uns nur daran zu erinnern, daß bei Zusatz einer starken Base zu einem Ammoniumsalz Doppelzersetzung eintritt, so daB wir es zum Schlub nicht nur mit den Wirkungen eines Ammoniumsalzes auf die Fibrinquellung in starkem Alkali, sondern vielmehr weitgehend mit der Wirkung eines Natriumsalzes auf die Quellung von Fibrin in Ammoniumhydroxyd zú tun haben. Aehnliche ungewöhnliche Fälle finden wir auch bei Säuren, z. B. bei der Wirkung der Chloride des Bariums, Kalziums und Strontiums zusammen mit Schwefelsäure, nur hier mit unerwartet hohen Quellungswerten. 